\title{
Arthroderma benhamiae Infection in a Rabbit
}

\author{
Kumiko SAITO $^{1)}$, Rui KANO ${ }^{1) *}$, Yuka NAKAMURA ${ }^{2)}$, Shinichi WATANABE ${ }^{2)}$ and Atsuhiko HASEGAWA ${ }^{1)}$ \\ ${ }^{1)}$ Department of Pathobiology, Nihon University School of Veterinary Medicine, Fujisawa, Kanagawa, 252-8510 and ${ }^{2)}$ Department of \\ Dermatology, Teikyo University School of Medicine, Itabashi-ku, Tokyo 173-8605, Japn
}

(Received 21 November 2000/Accepted 18 April 2001)

ABSTRACT. The isolate from the rabbit with dermatophytosis which was transmitted to the owners was proved to be Arthroderma benhamiae (-) by mating experiments as well as by chitin synthase 1 (CHS1) gene analysis. KEY WORDS: Arthroderma benhamiae, chitin synthase 1 gene, rabbit.

Rabbit dermatophytoses have been reported mainly in laboratory rabbit colonies and rabbits for commercial breeding $[1,3,8]$ and were caused mostly by Trichophyton mentagrophytes. Although dermatophytosis is one of important zoonoses, there are few reports describing human cases transmitted from rabbits. Today many rabbits share the enviroment with the owners as companion animals. Therefore, it is important to diagnose a rabbit dermatophytosis correctly in small animal clinics as well as on the viewpoint of public health.

T. mentagrophytes was confirmed to be a complex including at least 3 species; Arthroderma benhamiae, A. simii and A. vanbreuseghemii $[1,7,8,9]$. However, it is difficult to identify $T$. mentagrophytes isolates at the teleomorphic state by routine mycological examination. Recently molecular analysis was shown to be useful to differentiate these 3 species [5], indicating that some clinical isolates of T. mentagrophytes from human and animal dermatophytoses could be determined by molecular analysis [5]. In 1998, the first isolate of Arthroderma benhamiae in Japan from a 2-month-old rabbit was reported [6]. A. benhamiae was reported at first in U.S.A. and then isolated in Europe and Africa [1]. We report a rabbit case infected with A. benhamiae, which was investigated by molecular analysis as well as mycological examination.

January the 15th, 2000, a 4-month-old female dwarf rabbit was referred to the animal hospital in Urawa, Saitama, Japan. The chief complaint was hair loss, which had started several weeks before and had been gradually expanding. The rabbit had been kept for 3 months by the married couple. Physical examination revealed no abnormality except skin lesions, such as alopecia with scaly erytrema on the face, head, auricles, dorsal neck and hind legs (Fig. 1) and reddish swelling at the external genital area. The rabbit owners also had skin lesions on their hands, legs, face or axilla.

Based on the clinical signs, skin lesions of the rabbit were suspected to be caused by a dermatophyte and the symptoms around the mouth and the external genital area suggested

\footnotetext{
* CorRespondence to: Department of Pathobiology, Nihon University School of Veterinary Medicine, 1866 Kameino, Fujisawa, Kanagawa 252-8510, Japan,
}

Treponema infection. The specimens were collected from the lesions for the microscopic examination. The hyphae and arthroconidia of a dermatophyte were detected (Fig. 2), but spirocheta could not. Oral administration of griseofulvin $(25 \mathrm{mg} / \mathrm{kg}$, sid) and topical administration of ketoconazole cream $(1 \%)$ were carried out for dermatophytosis [2,3]. Chloramphenicol palmitate $(50 \mathrm{mg} / \mathrm{kg}$ bid p.o.) was also used.

After 4-week treatments, lesions on the face and genital area were cured and lesions on the other areas were also partially improved. Administration of chloramphenicol palmitate was continued for 6 weeks. Ten weeks after starting medication, the rabbit showed recovery almost all over the body except for auricles.

From skin scrapings, colonies develoned on DTM (dermatophyte test medium) and 1/10 diluted Sabrouraud's dextrose agar. The colony of this clinical isolate grew a white and powdery surface after 2 week-incubation on sunflower seed agar at $24^{\circ} \mathrm{C}$. Many tear-shaped microconidia and well-developed spirals were observed (Fig. 3). Therefore, the isolate was identified to be T. mentagrophytes. The same dermatophyte was isolated from skin lesions of the owners.

Isolates from the patient rabbit and the owners were examined by mating experiments as well as chitin synthase

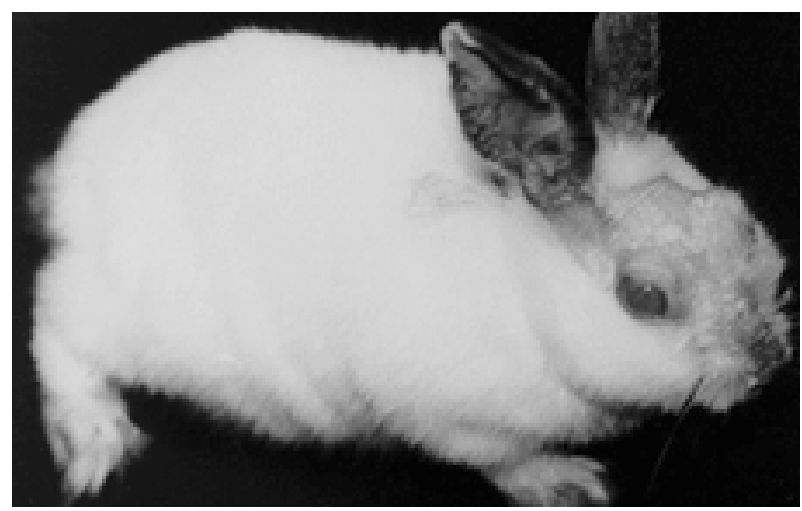

Fig. 1. The skin lesions showed alopecia and redness, with scales and scurfs on the rabbit face. 


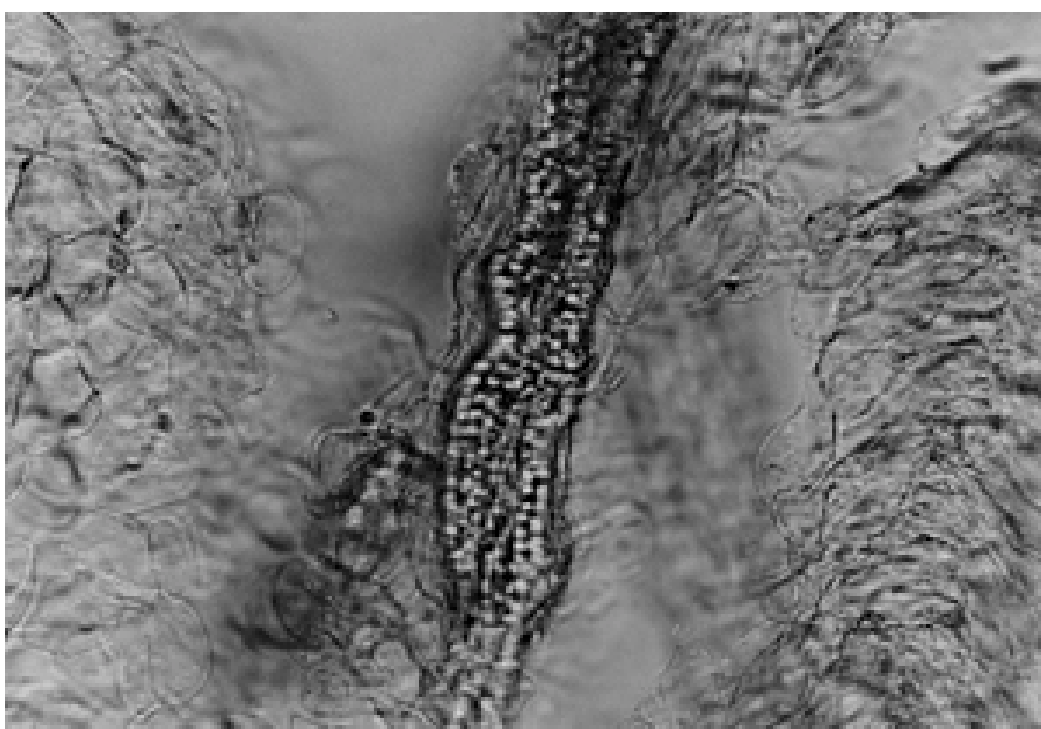

Fig. 2. Microscopic examination of skin scrapings from a skin lesion $(\times 400)$. Fungal hyphae and arthroconidia in skin scrapings from a skin lesion of the patient rabbit.

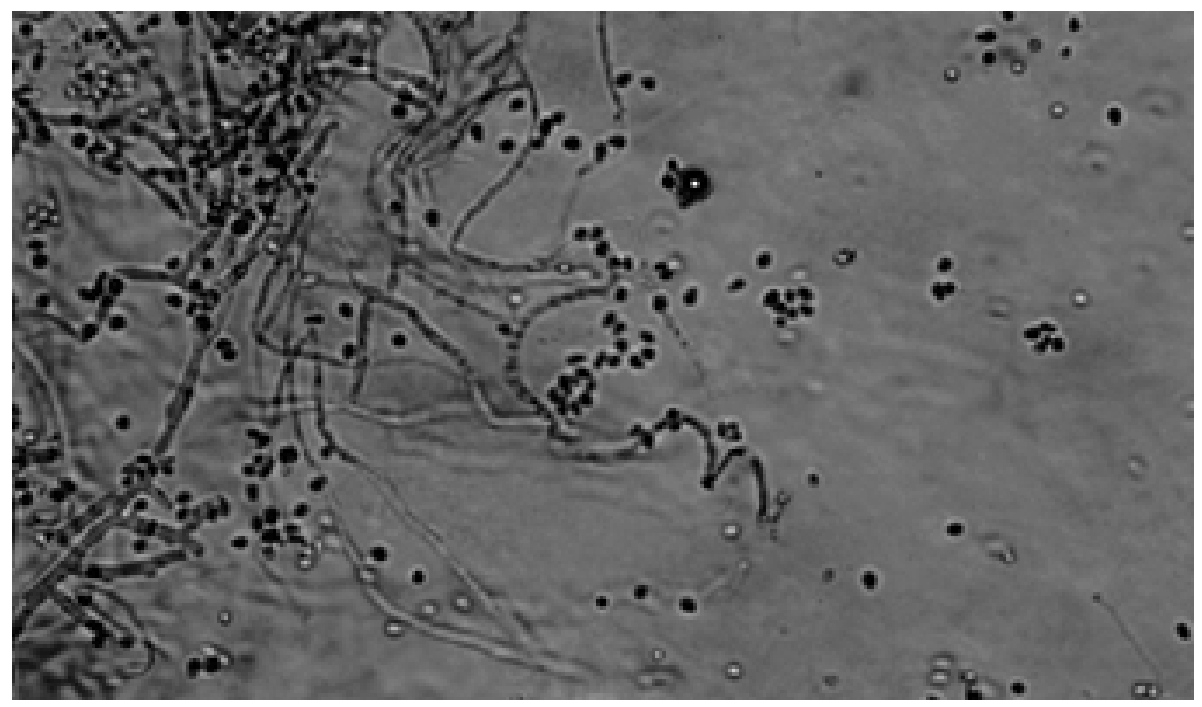

Fig. 3. Microconidia and spirals of the clinical isolate $(\times 400)$.

gene 1 analysis [5].

DNA from the clinical isolates from the rabbit and the owner were prepared by the method of Kano et al. [5, 6]. Briefly, the sequences of the degenerate primers for $\mathrm{CHS1}$ gene were based on the previous study reported [5]: primer 1, 5'-CTG AAG CTT ACT(ACG) ATG TAT(C) AAT(C) GAG(A) GAT(C)-3' and primer 2, 5'-GTT CTC GAG (C)TTT (A)GTA (C)TTC (A)GAA (A)GTT (T)CTG-3'. The PCR amplification of the clinical isolate DNA with degenerate $C H S 1$ primers yielded fragment of about $620 \mathrm{bp}$ and this fragments were cloned into plasmid vector and sequenced using an ABI PRISM 310 Genetic Analyzer (Perkin-Elmer, Foster City, U. S. A.).

Amplification of the clinical isolate DNA with degenerate $C H S 1$ primers yielded fragments of about $620 \mathrm{bp}$, consistent with the sizes of $C H S 1$ gene fragments from fungal species previously reported [5]. Nucleotide sequence analysis of the CHS1 gene fragments from the clinical isolates as well as tester strains of $A$. benhammiae (DDBJ data base AB003558), indicated that the sequence similarities were 99\% among them. Based on these results, it was concluded that the clinical isolate was identical to A. benhamiae. 
Table 1. Species and strains used in this study

\begin{tabular}{|c|c|}
\hline Species (Mating type) & Strain \\
\hline$A^{a)}$. benhamiae (+) & VUT $^{\text {b) }-77011 ~}$ \\
\hline A. benhamiae (-) & VUT-77012 \\
\hline A. $\operatorname{simii}(+)$ & VUT-77009 \\
\hline A. simii (-) & VUT-77010 \\
\hline A. vanbreuseghemii (+) & VUT-77007 \\
\hline A. vanbreuseghemii (-) & VUT-77008 \\
\hline$T^{c)}$ mentagrophytes & VUT-00003 (isolated from a rabbit) \\
\hline T. mentagrophytes & VUT-00003-2 (isolated from the owner) \\
\hline
\end{tabular}

a) Arthroderma, b) VUT: Veterinary Medicine, University of Tokyo, Japan, c) Trichophyton.

The mating experiments $[4,6]$ also confirmed those rabbit and human isolates to be A. benhamiae (-) mating type (Table 2).

Dermatophytoses due to T. mentagrophytes are sometimes transmited to pet owners from their animals. Each animal species appears to have different susceptibility to dermatophyte species. In human cases, T. mentagrophytes should be identified at a teleomorphic state, since it is important on the epidemiological points of view.

In this case, $A$. benhamiae was isolated as an etiologic dermatophyte from a patient rabbit and owner. A. benhamiae has been isolated from dermatophytosis of dog, horse, guinea pig and rabbit $[1,8]$. These animals exist close to human environment. Since this infection could be transmitted to a human from affected animals, a prudent care should be taken whenever handling infected animals.
Table 2. The result of mating experiments

\begin{tabular}{lcc}
\hline Species (mating type) strain & VUT-00003 & VUT-00003-2 \\
\hline$A^{a}$. benhamiae (+) VUT-77011 & $++^{\mathrm{b})}$ & + \\
A. benhamiae (-) VUT-77012 & - c) $^{\mathrm{c}}$ & - \\
A. simii (+) VUT=77009 & - & - \\
A. simii (-) VUT-77010 & - & - \\
A. vanbreuseghemii (+) VUT-77006 & - & - \\
A. vanbreuseghemii (-) VUT-77001 & - & - \\
\hline
\end{tabular}

a) Arthroderma, b) +: Ascospores are present, c) -: Ascospores are absent.

\section{REFERENCES}

1. Ajello, L. and Cheng, S.-L. 1967. Saboraudia 4: 230-234.

2. Alteras, I. And Cojocaru, I. 1969. Mykosen 12: 543-544.

3. Franklin, C. L., Gibson, S. V., Caffrey, C. J., Wagner, J. E. and Steffen, E. K. 1991. J. Am. Vet. Med. Assoc. 198: 1625-1630.

4. Hironaga, M. and Watanabe, S. 1980. Mycologia 72: 11591170.

5. Kano, R., Nakamura, Y., Watari, T., Watanabe, S., Takahashi, H., Tsujimoto, H. and Hasegawa, A. 1997. Mycoses 40: 411414.

6. Kano, R., Nakamura, Y., Yasuda, K., Watari, T., Watanabe, S., Takahashi, H. and Hasegawa, A. 1998. Microbiol. Immunol. 42: $575-578$.

7. Stockdale, M. P., Mackenzie, W. R. D. and Austwick, K. C. P. 1967. Saboraudia 4: 112-123.

8. Takashio, M. 1974. Intern. J. Dermatol. 13: 94-101.

9. Takashio, M. 1977. pp. 271-276. In: Recent Advances in Medical and Veterinary Mycology (Iwata, K. ed.), University of Tokyo Press, Tokyo. 